\title{
Charm elliptic flow in relativistic heavy-ion collisions
}

\author{
Bin Zhang, ${ }_{1}^{1}$ Lie-Wen Chen, ${ }^{2}$ and C. M. Ko ${ }^{3}$ \\ ${ }^{1}$ Department of Chemistry and Physics, P.O. Box 419, Arkansas State University, State University, Arkansas 72467-0419, USA \\ ${ }^{2}$ Institute of Theoretical Physics, Shanghai Jiao Tong University, Shanghai 200240, China \\ ${ }^{3}$ Cyclotron Institute and Physics Department, Texas A EMM University, College Station, Texas 77843-3366, USA
}

(Received 18 February 2005; published 31 August 2005)

\begin{abstract}
Charm elliptic flow in heavy-ion collisions at the Relativistic Heavy Ion Collider is studied in a multiphase transport model. Assuming that the cross section for charm quark scattering with other light quarks is the same as that between light quarks, we find that both charm and light quark elliptic flows are sensitive to the value of the cross section. Compared to that of light quarks, the elliptic flow of charm quarks is smaller at low transverse momentum but approaches comparable values at high transverse momentum. Similar features are seen in the elliptic flow of charmed mesons as well as that of the electrons from their semileptonic decays when the charmed mesons are produced from quark coalescence during hadronization of the partonic matter. To describe the large electron elliptic flow observed in available experimental data requires a charm quark-scattering cross section that is much larger than that given by the perturbative quantum chromodynamics.
\end{abstract}

DOI: 10.1103/PhysRevC.72.024906

PACS number(s): 25.75.Ld, 24.10.Lx

\section{INTRODUCTION}

Because of their large masses, heavy quarks are produced very early in ultrarelativistic heavy-ion collisions through hard collisions between nucleons. Their initial momentum spectra can thus be described by the perturbative quantum chromodynamics (pQCD). How their final spectra deviate from the initial ones depends on their interactions in the initial partonic matter and the mechanism that converts them to hadrons as well as their subsequent interactions in the hadronic matter. Using two extreme scenarios for the charmed meson spectrum (i.e., pQCD without final-state interactions and completely thermalized hydrodynamics with transverse flow velocity field), it was, however, found in Ref. [1] that the transverse momentum spectra of the electrons from their decay are both consistent with that measured in $\mathrm{Au}+\mathrm{Au}$ collisions at the Relativistic Heavy Ion Collider (RHIC) [2]. However, the two scenarios give very different elliptic flows for charmed mesons as well as their decay electrons, when the hadronization of charm quarks is modeled by coalescence with light quarks [3]. The study of heavy flavor particle elliptic flow in ultrarelativistic heavy-ion collisions thus provides the possibility of probing the interactions of heavy quarks in the partonic matter formed in these collisions and their hadronization mechanism.

The study in Ref. [3] is schematic as the charm quark elliptic flow in the scenario of complete thermalization is assumed to the same as the fitted light quark elliptic flow from experiments. To understand quantitatively the effect of final-state interactions on the charmed meson spectrum and elliptic flow, we use in the present study a multiphase transport (AMPT) model that includes both initial partonic and final hadronic interactions as well as the transition between these two phases of matter. Assuming that the cross section for charm quark scattering with light quarks is the same as that between light quarks, we find that the charm quark elliptic flow is sensitive to the value of the cross section and exhibits a transverse momentum dependence that is very different from that of light quarks. Although the elliptic flow of charm quarks at low transverse momentum is much smaller than that of light quarks as expected from the mass ordering of particle elliptic flows predicted in the hydrodynamic model, the two become comparable at high transverse momentum. For final charmed hadrons and their decay electrons, their elliptic flows are found to follow closely that of charm quarks and thus carry information on the charm quark interactions in the initial partonic matter. Our results are similar to those found in Ref. [4] based on Molnar's parton cascade (MPC) model. Conversely, the value of the charmed meson elliptic flow from our study is much larger than that found in Ref. [5] based on the hadron-string dynamics (HSD) model, in which the initial dense matter consists of strings and hadrons instead of partons as in the AMPT and MPC models. The thermalization and elliptic flows of charm quarks in a hydrodynamically expanding quark-gluon plasma have also been studied in a Langevin model, and they are found to be sensitive to the charm quark diffusion coefficient [6]. We note that preliminary results from the present study have been reported in Ref. [7].

This article is organized as follows. In Sec. II, we give a brief review of the AMPT model and then extend it to include the dynamics of charm particles. Results from the AMPT model on both the transverse momentum spectra and elliptic flows of charm particles are presented in Sec. III. Finally, a summary is given in Sec. IV.

\section{THE AMPT MODEL}

\section{A. A brief review}

The AMPT model [8-13] is a hybrid model that uses minijet partons from hard processes and strings from soft processes in the heavy-ion jet interaction generator (HIJING) model [14] as the initial conditions for modeling heavy-ion collisions at ultrarelativistic energies. Because the initial energy density in $\mathrm{Au}+\mathrm{Au}$ collisions at RHIC is much larger than the critical 
energy density at which the hadronic matter to quark-gluon plasma transition would occur $[10,13,15]$, we use the version which allows the melting of initial excited strings into partons [16]. In this version, hadrons that would have been produced from the HIJING model are converted to valence quarks and/or antiquarks. Interactions among these partons are described by Zhang's parton cascade (ZPC) model [17]. At present, this model includes only parton-parton elastic scatterings with an in-medium cross section given by the perturbative QCD, i.e.,

$$
\frac{d \sigma_{p}}{d t}=\frac{9 \pi \alpha_{s}^{2}}{2}\left(1+\frac{\mu^{2}}{s}\right) \frac{1}{\left(t-\mu^{2}\right)^{2}},
$$

where $\alpha_{s}=0.47$ is the strong coupling constant and $s$ and $t$ are the usual Mandelstam variables. The effective screening mass $\mu$ depends on the temperature and density of the partonic matter but is taken as a parameter in ZPC for fixing the magnitude and angular distribution of parton-scattering cross section. Because no inelastic scatterings are included in the ZPC model, only quarks and antiquarks from the melted strings are present in the partonic matter. The species independence of the above cross section compensates, however, for the absence of gluons in the early stage.

After partons stop scattering, they are converted to hadrons using a coordinate space quark coalescence model (i.e., two nearest quark and antiquark are combined into mesons and three nearest quarks or antiquarks are combined into baryons or antibaryons that are closest to the invariant masses of these parton combinations). This coalescence model is somewhat different from the ones that are based on the overlap of the hadron quark wave functions with the quark distribution functions in the partonic matter and used extensively for studying the production of hadrons with intermediate transverse momenta [18-20]. The final-state scatterings of produced hadrons in the AMPT model are described by a relativistic transport (ART) model [21].

Using parton-scattering cross sections of $6-10 \mathrm{mb}$, the AMPT model with string melting is able to reproduce the centrality and transverse momentum (below $2 \mathrm{GeV} / \mathrm{c}$ ) dependence of hadron elliptic flows [16] and higher order anisotropic flows [22] as well as the pion interferometry [23] measured in $\mathrm{Au}+\mathrm{Au}$ collisions at $\sqrt{s}=130 \mathrm{~A} \mathrm{GeV}$ at RHIC [24-26]. It has also been used for studying the kaon interferometry [27] in these collisions as well as many other observables at $\sqrt{s}=200 A \mathrm{GeV}[13,28]$.

\section{B. Including charm quarks and charmed mesons}

To include charm particles in the AMPT model, we first generate the initial charm quark distributions using information obtained by the STAR collaboration from $d+\mathrm{Au}$ collisions at $\sqrt{s_{N N}}=200 \mathrm{GeV}$ [29-31]. Because final-state interactions are negligible in this collision, the measured $D$-meson spectrum is related via the binary collision scaling to that from the nucleon-nucleon collision at same energy. In panel (a) of Fig. 1, we show the extracted transverse momentum spectrum of $D^{0}$ mesons in the rapidity interval $|y| \leqslant 1$ that are reconstructed from either the $K \pi$ invariant mass (solid squares) or the $K \pi \rho$ invariant mass (open circles).

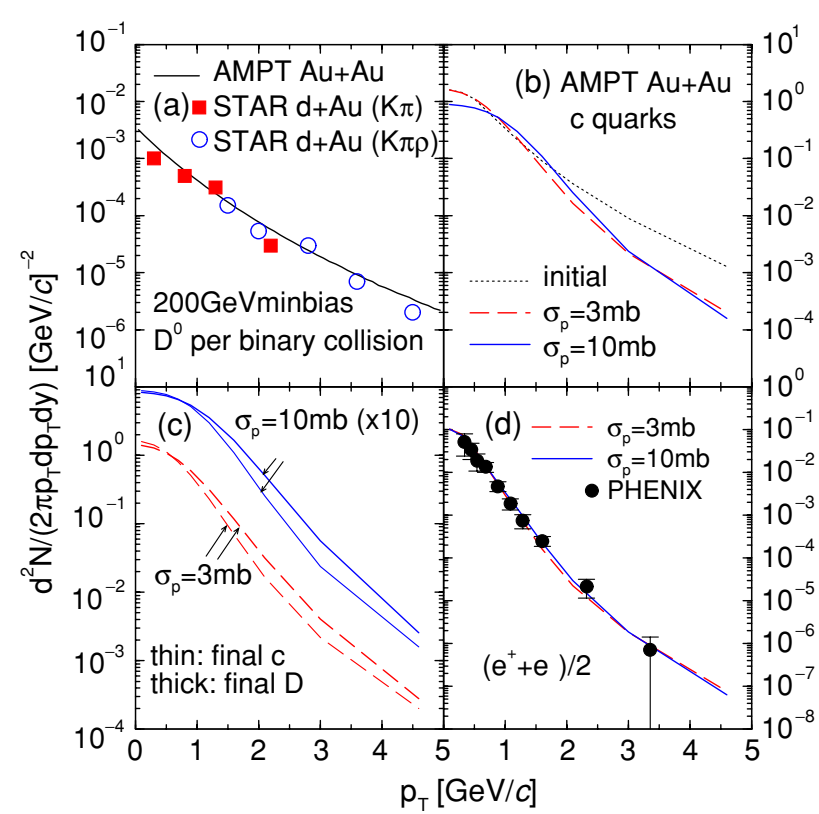

FIG. 1. (Color online) Transverse-momentum distributions at midrapidity in minimum bias $\mathrm{Au}+\mathrm{Au}$ collisions at $\sqrt{s_{N N}}=$ $200 \mathrm{GeV}$ : (a) $D^{0}$ mesons per binary nucleon-nucleon collision extracted from $d+\mathrm{Au}$ collisions in the STAR experiment [30] with solid squares and open circles denoting data and the solid curve from the parametrization [Eq. (2)]; (b) initial (dotted curve) and final (dashed curve for $\sigma_{p}=3 \mathrm{mb}$ and solid curve for $\sigma_{p}=10 \mathrm{mb}$ ) charm quarks from AMPT; (c) final charm quarks (thin dashed and solid curves) and $D$ mesons (thick dashed and solid curves); and (d) electrons from $D$-meson decay from AMPT (dashed and solid curves) and the PHENIX experiment [37] (solid circles).

Following Refs. [5,29-31], we parametrize this spectrum by a power law,

$$
\frac{d^{2} N}{2 \pi p_{T} d p_{T} d y} \propto\left(1+\frac{p_{T}}{3.25(\mathrm{GeV} / c)}\right)^{-8.0},
$$

as shown by the solid curve in Fig. 1(a). The positions of these charmed mesons are then distributed according to those of initial binary nucleon-nucleon collisions.

As for light hadrons produced from string fragmentation in the string melting scenario of the AMPT model, we dissociate these $D$ mesons into charm and anticharm quarks according to their valence structures to obtain the initial charm quark distributions. The charm quarks then propagate along straightline trajectories from the positions where they are produced in nucleon-nucleon collisions for a duration given by a formation time that is taken to be the inverse of the $D$-meson transverse momentum.

Because the cross section for charmed meson production in a nucleon-nucleon collision extracted from the STAR $d+\mathrm{Au}$ data is about $1.3 \mathrm{mb}$ and is about a factor of 30 smaller than the nucleon-nucleon inelastic cross section $(\sim 40 \mathrm{mb})$, the number of charm particles produced in relativistic heavy-ion collisions is small. To increase their statistics in the Monte Carlo treatment of the AMPT model, we apply the perturbative method that have been extensively used in transport models for studying rare particle production in heavy-ion collisions 
at lower energies [32,33]. Specifically, we scale the charm production cross section by a factor that equals the ratio of the nucleon-nucleon inelastic cross section to that for charm production. Each charm particle thus carries a probability that equals the inverse of this scale factor. As experimental data indicate that charm production in heavy-ion collisions at RHIC is consistent with the binary collision scaling, we only allow charm quarks to be produced in initial nucleonnucleon collisions. Rescattering of the charm quarks in the initial partonic matter and that of resulting charmed mesons in later hadronic matter is, however, included through the AMPT model. Although momenta of these charm particles are modified after scattering with other more abundant particles, their effects on the latter are neglected because of the smaller number of charm particles in each event.

Both the bulk dynamics of initial partonic matter and the time evolution of charm quarks are then described by the ZPC model using current quark masses (i.e., $9.9 \mathrm{MeV}$ for the $d$ quark, $5.6 \mathrm{MeV}$ for the $u$ quark, $199 \mathrm{MeV}$ for the $s$ quark, and $1.35 \mathrm{GeV}$ for the $c$ quark). In this study, we use two values for the cross section: $\sigma_{p}=3 \mathrm{mb}$ estimated from the perturbative QCD and $\sigma_{p}=10 \mathrm{mb}$ for a strongly interacting QCD. Charm rescattering cross sections have been studied before in Refs. [34,35], and the 3-mb cross section gives an upper bound for the corresponding energy-dependent charmrescattering cross sections when singularities are regulated by the screening mass. We use, however, the energy independent cross section to study whether experimental data require a cross section much larger than the perturbative one. The differential cross section then follows Eq. (1) with $s$ replaced by the maximum squared momentum transfer for charm rescatterings. As for the hadronization of light quarks, charm quarks are converted to hadrons using the coordinate space quark coalescence model described in the previous section. Because of the perturbative method used for treating charm quarks, charmed mesons acquire same probabilities as those of their parent charm quarks. Although the scattering cross sections of $D$ mesons with other hadrons have been studied in an effective hadronic model [36], we use in the present study instead the same cross section as that for parton-parton scattering. We have found that hadronic scattering does not affect much the final charmed meson spectrum and elliptic flow in heavy-ion collisions at RHIC. To compare with measured electrons from charmed meson decays, we include $D$ mesons that are both directly produced as well as from the decay of $D^{*}$ mesons at freeze-out.

\section{RESULTS}

\section{A. Charm transverse-momentum spectra}

The results from the AMPT model on the transversemomentum distributions of charm particles at midrapidity from minimum bias $\mathrm{Au}+\mathrm{Au}$ collisions at $\sqrt{s_{N N}}=200 \mathrm{GeV}$ are shown in Fig. 1. Panel (b) shows the initial charm quark transverse-momentum spectrum (dotted curve) together with final ones obtained using charm quark-scattering cross sections of $3 \mathrm{mb}$ (dashed curve) and $10 \mathrm{mb}$ (solid curve). The initial charm quark transverse-momentum spectrum is softer than that of initial $D^{0}$ mesons shown in panel (a), and this is because of the effective quenching introduced by converting $D$ mesons into their valence quarks. Partonic scattering leads to further quenching of charm quark spectrum, with the effect increasing with increasing charm quark-scattering cross section. The quenching effect is, however, reversed when charm quarks and light quarks are recombined into $D$ mesons via the coalescence model as shown in Fig. 1(c), where the charm quark spectra at the end of partonic phase (thin curves) and the $D$-meson spectra at the end of hadronic phase (thick curves) are compared. We note that the final $D$-meson number in midrapidity is about $20 \%$ larger than that of charm quarks in the same rapidity, and they are produced from charm quarks at larger rapidity as a result of the coordinate space coalescence.

Although the $D$-meson spectrum in $\mathrm{Au}+\mathrm{Au}$ collisions has not been measured at RHIC, that of the electrons from $D$-meson semileptonic decay is available from the PHENIX collaboration [37] as shown by solid circles in panel (d) of Fig. 1. They are very well described by the AMPT model with both charm quark-scattering cross sections of $3 \mathrm{mb}$ (dashed curve) and $10 \mathrm{mb}$ (solid curve). Similar to that found in Ref. [1], these experimental data thus cannot distinguish the dynamics of charm quarks in the partonic matter. This is not surprising as the decay process significantly softens the transverse-momentum spectrum of electrons with respect to that of $D$ mesons. We note that in this comparison the charm production cross section in a nucleon-nucleon collision is taken to be $600 \mu \mathrm{b}$ to be consistent with the PHENIX measurement.

\section{B. Charm elliptic flow}

We have also studied the elliptic flows of charm quarks and $D$ mesons, i.e., the second Fourier coefficient $v_{2}$ in the decomposition of their transverse-momentum distributions with respect to the azimuthal angle $\phi$ in the reaction plane [38],

$$
E \frac{d^{3} N}{d p^{3}}=\frac{1}{2 \pi} \frac{d N}{p_{T} d p_{T} d y}\left[1+\sum_{n=1}^{\infty} 2 v_{n}\left(p_{T}, y\right) \cos (n \phi)\right] .
$$

Because of the symmetry $\phi \leftrightarrow-\phi$ in the collision geometry, no sine terms appear in the above expansion. The anisotropic flows $v_{n}$ generally depend on the particle transverse momentum and rapidity, and for a given rapidity the anisotropic flows at transverse momentum $p_{T}$ can be evaluated from the following:

$$
v_{n}\left(p_{T}\right)=\langle\cos (n \phi)\rangle,
$$

where $\langle\cdots\rangle$ denotes average over the azimuthal distribution of particles with transverse momentum $p_{T}$. The elliptic flow $v_{2}$ can further be expressed in terms of the single-particle average:

$$
v_{2}\left(p_{T}\right)=\left\langle\frac{p_{x}^{2}-p_{y}^{2}}{p_{T}^{2}}\right\rangle,
$$

where $p_{x}$ and $p_{y}$ are, respectively, projections of particle momentum in and perpendicular to the reaction plane. It has been shown that the elliptic flow is sensitive to the early dynamics of produced matter in relativistic heavy-ion collisions [39-44], and it is thus an especially robust observable for studying the 


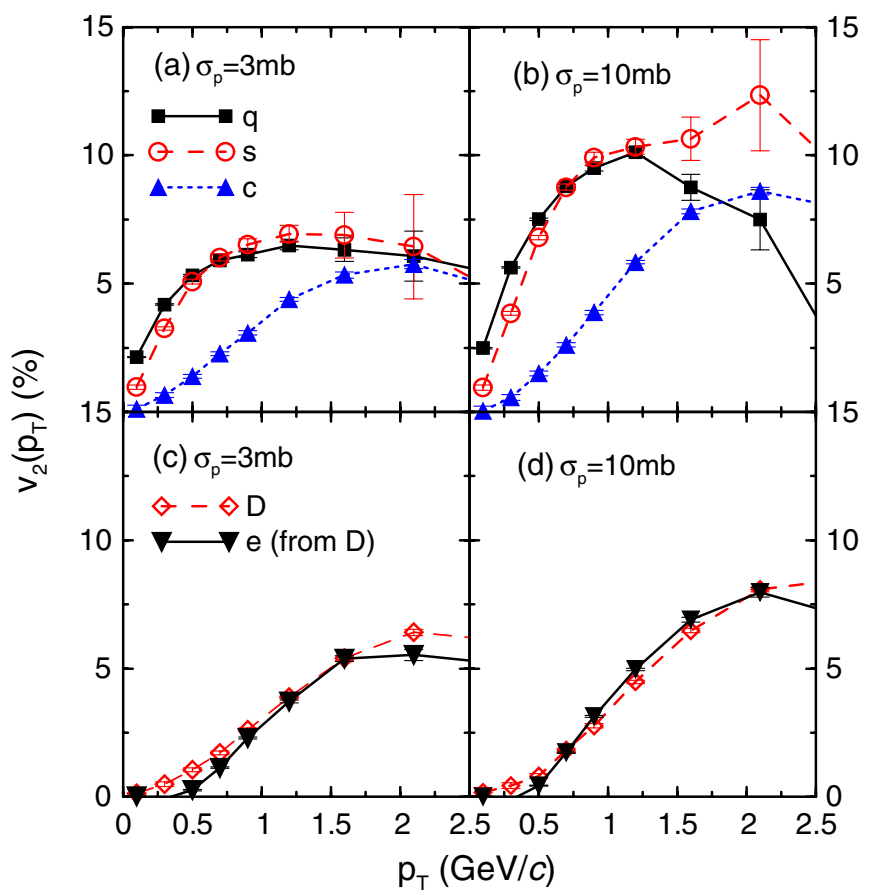

FIG. 2. (Color online) Elliptic flows of light, strange, and charm quarks (upper panels), $D$ mesons, and their decay electrons (lower panels) in minimum bias $\mathrm{Au}+\mathrm{Au}$ collisions at $\sqrt{s_{N N}}=200 \mathrm{GeV}$ for parton-scattering cross sections of $\sigma_{p}=3 \mathrm{mb}$ (left panels) and $\sigma_{p}=10 \mathrm{mb}$ (right panels).

interactions of early produced particles like charm quarks in the partonic matter.

In Fig. 2, we show the transverse-momentum dependence of elliptic flows of light, strange, and charm quarks as well as those of $D$ mesons and electrons from their decays in minimum bias $\mathrm{Au}+\mathrm{Au}$ collisions at $\sqrt{s_{N N}}=200 \mathrm{GeV}$. As shown in the upper panels (a) and (b), the $v_{2}$ displays a clear mass dependence, with the lighter quark having a larger value of $v_{2}$ at low $p_{T}$ and reaching the maximum value at a smaller $p_{T}$. This mass effect is particularly strong between the heavy charm quark and other light quarks. However, the maximum value of $v_{2}$ is similar for all quarks, exhibiting thus a very weak quark mass dependence. It is, however, sensitive to the partonscattering cross section, increasing from about $6 \%$ to about $9 \%$ when the parton-scattering cross-section changes from $\sigma_{p}=$ 3 to $10 \mathrm{mb}$. The mass dependence of quark $v_{2}$ at lower $p_{T}$ is similar to the mass ordering of particle elliptic flows observed in hydrodynamic models. Our results thus imply that with a parton-scattering cross section larger than $\sigma_{p}=3$ both low $p_{T}$ charm quarks and light quarks probably approach thermal equilibrium in heavy-ion collisions at RHIC.

From the lower panels of Fig. 2, it is seen that the $D$-meson elliptic flows (open diamonds) follow closely corresponding charm quark elliptic flows shown in the upper panels of Fig. 2, although they are slightly shifted to higher $p_{T}$ and also have slightly higher values relative to those of charm quarks. Also shown in the lower panels of Fig. 2 are the elliptic flows of electrons from $D$-meson decays (solid triangles), and they also follow closely the corresponding $D$-meson ones.

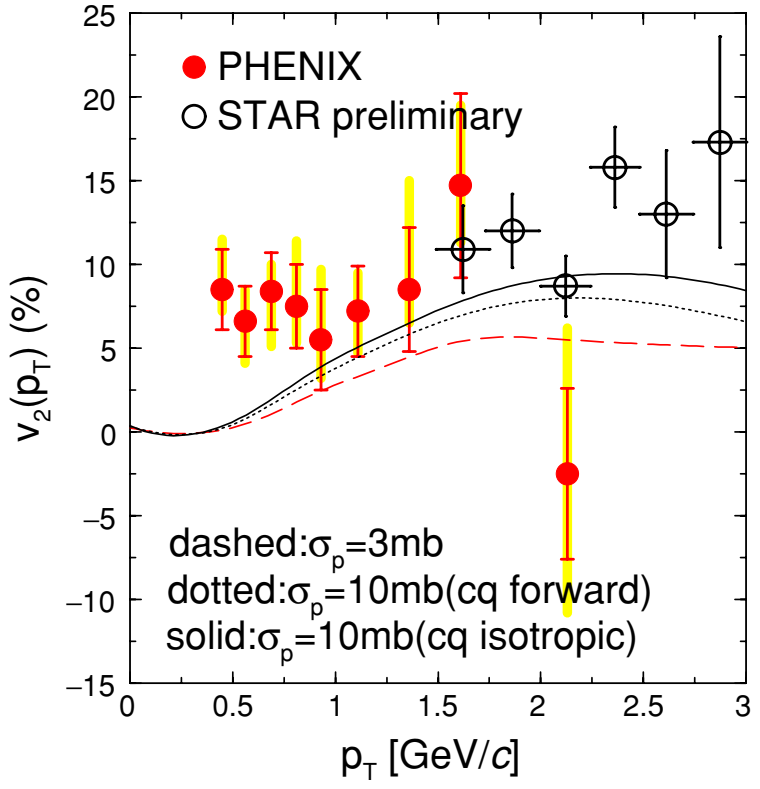

FIG. 3. (Color online) Transverse-momentum dependence of the elliptic flows of electrons from $D$-meson decays from the AMPT model for different parton scattering cross sections of 3 (dashed curve) and 10 (dotted curve) mb with angular distribution given by Eq. (1) as well as $10 \mathrm{mb}$ with isotropic angular distribution (solid curve) from minimum-bias $\mathrm{Au}+\mathrm{Au}$ collisions at $\sqrt{s_{N N}}=200 \mathrm{GeV}$. Available experimental data are from the PHENIX [45] (solid circles) and STAR [46] (open circles) collaborations.

It is interesting to see that at around $p_{T}=300 \mathrm{MeV}$, the electron elliptic flow becomes slightly negative, indicating that on average these electrons are moving perpendicular to their parent $D$ mesons. At $p_{T}$ above $2 \mathrm{GeV}$, the electron elliptic flow is slightly smaller than the $D$-meson elliptic flow. Although the electron $p_{T}$ differential elliptic flow is almost identical to that of $D$ mesons, the integrated electron elliptic flow is much smaller than that of $D$ mesons as the electron transverse spectrum is much softer than that of $D$ mesons. We find, e.g., $v_{2 e}=0.55 \pm 0.01 \% \ll v_{2 D}=2.73 \pm 0.04 \%$ and $v_{2 e}=0.90 \pm 0.01 \% \ll v_{2 D}=3.91 \pm 0.04 \%$ for parton cross sections of 3 and $10 \mathrm{mb}$, respectively.

In Fig. 3, we compare the elliptic flow of electrons from $D$-meson decay obtained from the AMPT model with available experimental data from the PHENIX [45] (solid circles) and STAR [46] (open circles) collaborations. The theoretical results are shown by the dashed and dotted curves for charm-scattering cross sections of 3 and $10 \mathrm{mb}$, respectively. Compared with the experimental data, the calculated charm flow has a similar magnitude at $1<p_{T}<1.5 \mathrm{GeV} / c$ but is somewhat smaller at other transverse momenta.

In Ref. [3], the elliptic flow of electrons from $D$-meson decay has also been studied in the coalescence model based on charm and light quark elliptic flows that are similar to ours shown in Fig. 2, and its value at high transverse momentum is larger and thus closer to the experimental data. The smaller charm elliptic flow from our study is partially because of the quark spatial anisotropy, particularly the negative spatial eccentricity $s_{2}$, that is present in the dynamical AMPT model 
but is neglected in Ref. [3]. As shown in Ref. [47], a negative quark $s_{2}$ reduces the elliptic flow of produced hadrons in a dynamic coalescence model such as the one used in the AMPT model.

As mentioned in Sec. II, the magnitude of parton-scattering cross sections used in the AMPT model is determined by the screening mass or parameter $\mu$ shown in Eq.(1). Because the larger cross section $\sigma_{p}=10 \mathrm{mb}$ is obtained by using a smaller value for $\mu$, its angular distribution becomes more forward peaked than in the case of $3 \mathrm{mb}$, thus reducing the effect of increasing cross section on the transport properties of quarks. However, it has recently been suggested that the scattering between charm and light quarks might go through a quasicolorless charmed resonance, leading to a cross section that is not only large but also isotropic [48]. To see the effect of such a scattering mechanism, we have carried out a calculation with an isotropic charmlight-quark-scattering cross section of $10 \mathrm{mb}$, and the elliptic flow of electrons from the decay of resulting $D$ mesons is shown by the solid curve in Fig. 3. It is seen that this indeed further enhances the charm elliptic flow but not enough to reproduce available experimental data. If the charm elliptic flow remains large when more accurate data are available, then the charm quark-scattering cross section will be even larger than what have been used in present study.

We note that a naive or additive quark coalescence model $[49,50]$, which ignores the effect of momentum distribution of quarks inside hadrons [51,52], the nonnegligible local spatial anisotropy [47], and the large local directed flow [53], has been sometimes used for calculating the hadron elliptic flows from those of quarks. In this case, the elliptic flows of hadrons are then simple sums of the elliptic flows of their constituent quarks. Using this simplified quark coalescence model, the elliptic flow of electrons from $D$ mesons produced from the charm and light quark elliptic flows shown in Fig. 2 for a parton-scattering cross section of $10 \mathrm{mb}$ is comparable to that seen in available experimental data.

\section{SUMMARY}

We have studied the transverse-momentum spectra and elliptic flows of charm particles and the electrons from charmed meson decay in heavy-ion collisions at RHIC in the AMPT model by treating the screening mass in the in-medium pQCD scattering cross section between light quarks as a parameter and assuming that charm quarks have similar cross sections.
Using an initial charm quark transverse-momentum spectrum obtained from the dissociation of the empirically determined charmed meson spectrum in nucleon-nucleon collisions at same energy, we find that the final transverse-momentum spectrum is softer than that in nucleon-nucleon collisions as a result of the quenching effect because of charm quark rescattering in the partonic matter. Although the quenching effect increases with increasing charm quark scattering cross section, the transverse momentum spectrum of electrons from resulting $D$-meson decays does not depend much on the parton scattering cross sections as a result of the stronger quenching effect because of the decay process, and results using both cross sections of 3 and $10 \mathrm{mb}$ are consistent with available experimental data from the PHENIX experiment.

The elliptic flow of charm quarks is, however, sensitive to the parton cross section as that of light quarks. With respect to the light quark elliptic flow, the charm quark elliptic flow is smaller at low transverse momentum but reaches a similar value at high transverse momentum. With the coordinate space quark coalescence model for hadronization in the AMPT model, the elliptic flow of $D$ mesons is found to follow that of charm quarks. Compared to measured elliptic flow of electrons from $D$-meson decay in $\mathrm{Au}+\mathrm{Au}$ collisions at $\sqrt{s}=200 \mathrm{~A} \mathrm{GeV}$, the calculated values from the AMPT model, which is close to the $D$-meson elliptic flow, are somewhat smaller even for an isotropic charmlight-quark-scattering cross section of $10 \mathrm{mb}$. Such a cross section is much larger than that given by the perturbative QCD estimate but is consistent with that based on novel resonant heavy-light quark interactions [48] inside a strongly interacting quark-gluon plasma consisting of quasiparticles and colored bound states [54]. The study of charm flow in relativistic heavy-ion collisions thus provides a promising tool to explore the properties of produced partonic matter and its hadronization dynamics.

\section{ACKNOWLEDGMENTS}

We thank Vincenzo Greco, Miklos Gyulassy, Zi-Wei Lin, Scott Pratt, Ralf Rapp, and An Tai for useful discussions. This article was based on work supported by the U.S. National Science Foundation under grants PHY-0098805 and PHY-0457265 (C.M.K.) and PHY-0140046 (B.Z.), the Welch Foundation under grant A-1358 (C.M.K.), and the National Natural Science Foundation of China under grant 10105008 (L.W.C.).
[1] S. Batsouli, S. Kelly, M. Gyulassy, and J. L. Nagle, Phys. Lett. B557, 26 (2003).

[2] K. Adcox et al. (PHENIX Collaboration), Phys. Rev. Lett. 88, 192303 (2002); Nucl. Phys. A715, 695 (2003).

[3] V. Greco, C. M. Ko, and R. Rapp, Phys. Lett. B595, 202 (2004).

[4] D. Molnar, J. Phys. G 31, S421 (2005).

[5] E. L. Bratkovskaya, W. Cassing, H. Stocker, and N. Xu, Phys. Rev. C 71, 044901 (2005).

[6] G. D. Moore and D. Teaney, Phys. Rev. C 71, 064904 (2005).

[7] L. W. Chen and C. M. Ko, J. Phys. G 31, S49 (2005).
[8] B. Zhang, C. M. Ko, B. A. Li, and Z. Lin, Phys. Rev. C 61, 067901 (2000).

[9] Z. W. Lin, S. Pal, C. M. Ko, B. A. Li, and B. Zhang, Phys. Rev. C 64, 011902(R) (2001); Nucl. Phys. A698, 375 (2002).

[10] B. Zhang, C. M. Ko, B. A. Li, Z. W. Lin, and B. H. Sa, Phys. Rev. C 62, 054905 (2000); B. Zhang, C. M. Ko, B. A. Li, Z. Lin, and S. Pal, ibid. 65, 054909 (2002).

[11] C. M. Ko, Z. W. Lin, and S. Pal, Heavy Ion Phys. 17, 219 (2003).

[12] S. Pal, C. M. Ko, and Z. W. Lin, Nucl. Phys. A730, 143 (2004). 
[13] Z. W. Lin, C. M. Ko, B. A. Li, B. Zhang, and S. Pal, arXiv:nuclth/0411110.

[14] X. N. Wang and M. Gyulassy, Phys. Rev. D 44, 3501 (1991).

[15] D. Kharzeev and M. Nardi, Phys. Lett. B507, 121 (2001).

[16] Z. W. Lin and C. M. Ko, Phys. Rev. C 65, 034904 (2002).

[17] B. Zhang, Comput. Phys. Commun. 109, 193 (1998).

[18] V. Greco, C. M. Ko, and P. Lévai, Phys. Rev. Lett. 90, 202302 (2003); Phys. Rev. C 68, 034904 (2003).

[19] R. C. Hwa and C. B. Yang, Phys. Rev. C 67, 034902 (2003); 67, 064902 (2003).

[20] R. J. Fries, B. Müller, C. Nonaka, and S. A. Bass, Phys. Rev. Lett. 90, 202303 (2003); Phys. Rev. C 68, 044902 (2003).

[21] B. A. Li and C. M. Ko, Phys. Rev. C 52, 2037 (1995); B. A. Li, A. T. Sustich, B. Zhang, and C. M. Ko, Int. J. Phys. E 10, 267 (2001).

[22] L. W. Chen, C. M. Ko, and Z. W. Lin, Phys. Rev. C 69, 031901(R) (2004).

[23] Z. W. Lin, C. M. Ko, and S. Pal, Phys. Rev. Lett. 89, 152301 (2002).

[24] K. H. Ackermann et al. (STAR Collaboration), Phys. Rev. Lett. 86, 402 (2001).

[25] J. Adams et al. (STAR Collaboration), Phys. Rev. Lett. 92, 062301 (2004).

[26] C. Adler et al. (STAR Collaboration), Phys. Rev. Lett. 87, 082301 (2001).

[27] Z. W. Lin and C. M. Ko, J. Phys. G 30, S263 (2004).

[28] L. W. Chen, V. Greco, C. M. Ko, and P. F. Kolb, Phys. Lett. B605, 95 (2005).

[29] J. Adams et al. (STAR Collaboration), Phys. Rev. Lett. 94, 062301 (2005).

[30] A. Tai (STAR Collaboration), J. Phys. G 30, S809 (2004).

[31] L. Ruan (STAR Collaboration), J. Phys. G 30, S1197 (2004).
[32] J. Randrup and C. M. Ko, Nucl. Phys. A343, 519 (1980).

[33] X. S. Fang, C. M. Ko, and Y. M. Zheng, Nucl. Phys. A556, 499 (1993).

[34] B. L. Combridge, Nucl. Phys. B151, 429 (1979).

[35] B. Svetitsky, Phys. Rev. D 37, 2484 (1988).

[36] Z. Lin, C. M. Ko, and B. Zhang, Phys. Rev. C 61, 024904 (2000); Z. W. Lin, T. G. Di, and C. M. Ko, Nucl. Phys. A689, 965 (2001).

[37] S. S. Adler et al. (PHENIX Collaboration), Phys. Rev. Lett. 94, 082301 (2005).

[38] A. M. Poskanzer and S. A. Voloshin, Phys. Rev. C 58, 1671 (1998).

[39] H. Sorge, Phys. Rev. Lett. 78, 2309 (1997).

[40] B. Zhang, M. Gyulassy, and C. M. Ko, Phys. Lett. B455, 45 (1999).

[41] P. F. Kolb, J. Sollfrank, and U. Heinz, Phys. Rev. C 62, 054909 (2000).

[42] D. Teaney, J. Lauret, and E. V. Shuryak, arXiv:nucl-th/0110037.

[43] D. Molnar and M. Gyulassy, Nucl. Phys. A697, 495 (2002); A703, 893 (2002).

[44] D. Molnar and P. Huovinen, Phys. Rev. Lett. 94, 012302 (2005).

[45] S. S. Adler et al. (PHENIX Collaboration), Phys. Rev. C 72, 024901 (2005).

[46] F. Laue (STAR Collaboration), J. Phys. G 31, S27 (2005).

[47] S. Pratt and S. Pal, Phys. Rev. C 71, 014905 (2005).

[48] H. van Hees and R. Rapp, Phys. Rev. C 71, 034907 (2005).

[49] P. F. Kolb, L. W. Chen, V. Greco, and C. M. Ko, Phys. Rev. C 69, 051901(R) (2004).

[50] D. Molnar and S. A. Voloshin, Phys. Rev. Lett. 91, 092301 (2003).

[51] Z. W. Lin and D. Molnar, Phys. Rev. C 68, 044901 (2003).

[52] V. Greco and C. M. Ko, Phys. Rev. C 70, 024901 (2004).

[53] D. Molnar, arXiv:nucl-th/0408044.

[54] E. V. Shuryak and I. Zahed, Phys. Rev. C 70, 021901(R) (2004); Phys. Rev. D 70, 054507 (2004). 